MATHEMATICS OF COMPUTATION

Volume 68, Number 228, Pages 1521-1531

S $0025-5718(99) 01102-3$

Article electronically published on March 4, 1999

\title{
MESHLESS GALERKIN METHODS USING RADIAL BASIS FUNCTIONS
}

\author{
HOLGER WENDLAND
}

\begin{abstract}
We combine the theory of radial basis functions with the field of Galerkin methods to solve partial differential equations. After a general description of the method we show convergence and derive error estimates for smooth problems in arbitrary dimensions.
\end{abstract}

\section{INTRODUCTION}

Interpolation by radial basis functions has become a powerful tool in multivariate approximation theory, especially since compactly supported radial basis functions are available. We shall collect the necessary results in the third section, but refer the reader to the survey articles $[6,8,10,11]$ for details.

In this paper we describe how radial basis functions can be used to solve elliptic partial differential equations numerically. We choose the same Galerkin approach as in classical finite element methods. The results presented here are comparable to those of classical FEM.

Since, in contrast to FEM, the effort for the construction of the finite dimensional subspace using radial basis functions is independent of the current space dimension, it is in principle possible to solve high dimensional problems as they occur in quantum mechanics (cf. [9]). For example, the $n$-body problem of $n$ interacting particles leads in the stationary case to a time-independent Schrödinger equation on $\mathbb{R}^{3 n}$. Under certain additional conditions on the potential it is possible to approximate the solution of this global problem by a solution of a boundary value problem on a finite domain.

But even in two or three space dimensions it could be reasonable to use our method: Classical finite element methods spend a lot of time on technical details concerning the mesh, especially for time-dependent problems with moving boundaries. The mesh has to be generated, adapted to singularities of the solution, and adapted to the changes of the domain. Meshless methods don't need to handle such problems because they only use unrelated centers for the discretisation. See [2] for an overview of general meshless methods and applications in engineering. Finally, very smooth solutions can be constructed as simply as less smooth solutions.

In the next section we describe in more detail the partial differential equation we are interested in. We restrict ourselves to second order partial differential equations, but a generalization to higher order equations can be done in an obvious way. As a

Received by the editor April 1, 1997.

1991 Mathematics Subject Classification. Primary 35A40, 35J50, 41A25, 41A30, 41A63, $65 \mathrm{~N} 15,65 \mathrm{~N} 30$.

Key words and phrases. Approximation orders, positive definite functions, PDE. 
reference for finite element methods or elliptic partial differential equations we give [3].

In the third section we give a short summary of the theory of radial basis function interpolation. In the fourth section we show how this theory can be used for Galerkin or Rayleigh-Ritz approximation, and derive results concerning a special kind of basis function, which generates Sobolev space as its native space. In this situation our results are comparable to those of classical finite element methods.

In the last section we generalize these results to more general basis functions, which allows us to give approximation orders even if the exact smoothness of the solution is unknown.

\section{PDE AND GALERKIn METhods}

For a bounded domain $\Omega$ with $C^{1}$-boundary $\partial \Omega$ we consider problems of the form

$$
\begin{aligned}
-\sum_{i, j=1}^{d} \frac{\partial}{\partial x_{i}}\left(a_{i j} \frac{\partial u}{\partial x_{j}}\right)(x)+c(x) u(x) & =f(x), \quad x \in \Omega, \\
\sum_{i, j=1}^{d} a_{i j}(x) \frac{\partial u(x)}{\partial x_{j}} \nu_{i}(x)+h(x) u(x) & =g(x), \quad x \in \partial \Omega,
\end{aligned}
$$

where $a_{i j}, c \in L_{\infty}(\Omega), i, j=1 \ldots, n, f \in L_{2}(\Omega), a_{i j}, h \in L_{\infty}(\partial \Omega), g \in L_{2}(\partial \Omega)$ and $\nu$ denotes the unit normal vector to the boundary $\partial \Omega$. The matrix $A(x)=\left(a_{i j}(x)\right)$ is assumed to be uniformly elliptic on $\Omega$, i.e. there is a constant $\gamma$ such that for all $x \in \Omega$ and all $\alpha \in \mathbb{R}^{d}$

$$
\gamma \sum_{j=1}^{d} \alpha_{j}^{2} \leq \sum_{i, j=1}^{d} a_{i j}(x) \alpha_{i} \alpha_{j}
$$

We further require that $c \geq 0$ and $h \geq 0$, and that at least one of them is uniformly bounded away from zero on a subset of nonzero measure of $\Omega$ or $\partial \Omega$, respectively.

Under these asumptions the variational approach leads to the strictly coercive and continuous bilinear form

$$
a(u, v)=\int_{\Omega}\left(\sum_{i, j=1}^{d} a_{i j} \frac{\partial u}{\partial x_{j}} \frac{\partial v}{\partial x_{i}}+c u v\right) d x+\int_{\partial \Omega} h u v d S
$$

on $V \times V$ with $V=W_{2}^{1}(\Omega)$, and to the continuous linear form

$$
F(v)=\int_{\Omega} f v d x+\int_{\partial \Omega} g v d S .
$$

on $V=W_{2}^{1}(\Omega)$. The corresponding variational problem

$$
\text { find } u \in W_{2}^{1}(\Omega) \text { such that } a(u, v)=F(v) \text { for all } v \in W_{2}^{1}(\Omega)
$$

has a unique solution by the Lax-Milgram theory.

This approach allows us to work with the whole Sobolev space $W_{2}^{1}(\Omega)$ and does not restrict us to the subspace $\stackrel{\circ}{W}_{2}^{1}(\Omega)$ consisting of functions with zero boundary values that often occurs with problems with pure Dirichlet boundary values. The boundary conditions themselves are incorporated into the bilinear form $a$ and the linear form $F$. 
To solve (2.4) numerically, the Galerkin method starts with a finite dimensional subspace $V_{N}$ of $V$ and computes the solution of the discretization

$$
\text { find } u_{N} \in V_{N} \text { such that } a\left(u_{N}, v\right)=F(v) \text { for all } v \in V_{N} \text {. }
$$

The error between the solution $u$ of (2.4) and the numerical solution $u_{N}$ can be bounded via Cea's lemma, which is in this context given by

$$
\left\|u-u_{N}\right\|_{W_{2}^{1}(\Omega)} \leq C \inf _{v \in V_{N}}\|u-v\|_{W_{2}^{1}(\Omega)} .
$$

Here and in what follows, $C$ will denote a generic constant.

We shall require $u$ to be more regular than $u \in W_{2}^{1}(\Omega)$. More precisely, we need $u \in W_{2}^{k}(\Omega)$ with $k>\frac{d}{2}$ if $d$ is the current space dimension. This is, for instance, satisfied if the boundary of $\Omega$ and the given functions are sufficiently smooth.

\section{RADIAL BASIS FUnCTIONS}

In this paper we want to use finite dimensional subspaces $V_{N}$ of $V=W_{2}^{1}(\Omega)$ of the form

$$
V_{N}:=\operatorname{span}\left\{\Phi\left(\cdot-x_{1}\right), \ldots, \Phi\left(\cdot-x_{N}\right)\right\}+\mathbb{P}_{m}^{d}
$$

where $\Phi: \mathbb{R}^{d} \rightarrow \mathbb{R}$ is at least a $C^{1}$-function, $\mathbb{P}_{m}^{d}$ denotes the space of polynomials of degree less than $m$ and $X=\left\{x_{1}, \ldots, x_{N}\right\} \subseteq \Omega$ is a set of pairwise distinct centers. The most interesting case is when $\Phi$ is compactly supported and $m=0$, i.e. no polynomials are added. In this case the stiffness matrix

$$
a\left(\Phi\left(\cdot-x_{j}\right), \Phi\left(\cdot-x_{k}\right)\right)
$$

is sparse. Moreover, for a radially symmetric $L$ and a radial $\Phi$, i.e. $\Phi(x)=\phi\left(\|x\|_{2}\right)$, $x \in \mathbb{R}^{d}$, with a univariate function $\phi: \mathbb{R}_{\geq 0} \rightarrow \mathbb{R}$, most of the entries of the stiffness matrix can be easily computed (cf [13]).

We are now considering the approximation error determined by (2.6). Therefore we invoke the theory of radial basis functions.

Definition 3.1. A function $\Phi: \mathbb{R}^{d} \rightarrow \mathbb{R}$ is said to be conditionally positive definite of order $m$ iff for all sets $X=\left\{x_{1}, \ldots, x_{N}\right\} \subseteq \mathbb{R}^{d}$ consisting of pairwise distinct centers $x_{j}$ and all $\alpha \in \mathbb{R}^{N} \backslash\{0\}$ satisfying $\sum_{j=1}^{N} \alpha_{j} x_{j}^{p}=0,|p|<m, p \in \mathbb{N}_{0}^{d}$, the inequality

$$
\sum_{j, k=1}^{N} \alpha_{j} \alpha_{k} \Phi\left(x_{j}-x_{k}\right)>0
$$

is valid. A conditionally positive definite function of order 0 is called a positive definite function.

The (radial) basis function interpolant $s_{u}$ to a function $u \in C\left(\mathbb{R}^{d}\right)$ on a set of centers $X$ is given by

$$
s_{u}(x)=\sum_{j=1}^{N} \alpha_{j} \Phi\left(x-x_{j}\right)+p(x),
$$

where $p$ is a polynomial of degree less than $m$. By interpolation, $s_{u}$ has to satisfy $s_{u}\left(x_{j}\right)=u\left(x_{j}\right), 1 \leq j \leq N$. The additional degrees of freedom are bounded by the conditions $\sum_{j=1}^{N} \alpha_{j} p\left(x_{j}\right)=0$, where $p$ runs through a basis of $\mathbb{P}_{m}^{d}$. It can be shown that there always exists an $s_{u}$ satisfying the required conditions (cf. [7]). 
TABLE 1. Radial basis functions

\begin{tabular}{|l|l|c|c|}
\hline Name & $\Phi(x)=\phi(r), r=\|x\|_{2}$ & $m$ & $F(h)$ \\
\hline Thin plate splines & $\begin{array}{l}(-1)^{1+\mu / 2} r^{\mu} \log r, \quad \mu \in 2 \mathbb{N} \\
(-1)^{\lceil\mu / 2\rceil} r^{\mu}, \quad \mu \in \mathbb{R}_{>0} \backslash 2 \mathbb{N},\end{array}$ & $\begin{array}{c}\mu / 2+1 \\
\lceil\mu / 2\rceil\end{array}$ & $h^{\mu / 2}$ \\
\hline Sobolev splines & $\begin{array}{l}K_{\mu-d / 2}(r) r^{\mu-d / 2}, \quad \mu>\frac{d}{2} \\
K \text { MacDonald's function }\end{array}$ & 0 & $h^{\mu-d / 2}$ \\
\hline $\begin{array}{l}\text { Compactly supported } \\
\text { functions, } C^{2 \ell}\end{array}$ & $\begin{array}{l}(1-r)_{+}^{\mu} p(r), \quad p \text { polynomial } \\
\partial p=\ell, \quad \mu=\lfloor d / 2\rfloor+2 \ell+1\end{array}$ & 0 & $h^{\ell+1 / 2}$ \\
\hline
\end{tabular}

Knowing that interpolation is always possible, we turn to the error analysis. Therefore we assume that the function $\Phi$ possesses a (generalized) Fourier transform $\widehat{\Phi}$ which is positive almost everywhere. This is satisfied for all common basis functions. We now introduce the native space $\mathcal{F}_{\Phi}$ consisting of all functions $f: \mathbb{R}^{d} \rightarrow \mathbb{R}$ which can be recovered via

$$
f(x)=(2 \pi)^{-d} \int_{\mathbb{R}^{d}} \widehat{f}(\omega) e^{i x^{T} \omega} d \omega,
$$

where $\widehat{f}$ is a function satisfying

$$
\widehat{f} / \sqrt{\widehat{\Phi}} \in L_{2}\left(\mathbb{R}^{d}\right) .
$$

The space $\mathcal{F}_{\Phi}$ possesses the semi-norm

$$
|f|_{\Phi}^{2}:=(2 \pi)^{-d} \int_{\mathbb{R}^{d}} \frac{|\widehat{f}(\omega)|^{2}}{\widehat{\Phi}(\omega)} d \omega
$$

with the nullspace $\mathbb{P}_{m}^{d}$. Thus $|\cdot|_{\Phi}$ is a norm if $\Phi$ is positive definite. In this case $\mathcal{F}_{\Phi}$ is a Hilbert space. If $\Phi$ is conditionally positive definite of order $m>0$, then the space $\mathcal{F}_{\Phi} / \mathbb{P}_{m}^{d}$ is a Hilbert space. For functions $u \in \mathcal{F}_{\Phi}$ it is possible to bound the error by

$$
\left|u(x)-s_{u}(x)\right| \leq P_{X, \Phi}(x)|u|_{\Phi}
$$

with the so-called Power function $P_{X, \Phi}(x)$ defined pointwise as the norm of the error functional. This Power function can be bounded in terms of the local data density given by $h_{\rho}(x):=\sup _{\|y-x\|_{2} \leq \rho} \min _{1 \leq j \leq N}\left\|y-x_{j}\right\|_{2}, \rho>0$ (cf. [17]). But if we restrict ourselves to basis functions having an algebraically decaying (generalized) Fourier transform, the proofs given in [17] allow us to choose $X \subseteq \Omega$ and to bound the Power function also in terms of the global data density

$$
h=h_{X, \Omega}:=\sup _{x \in \Omega} \min _{1 \leq j \leq N}\left\|x-x_{j}\right\|_{2},
$$

as long as $\Omega$ satisfies a uniform interior cone condition. In this case the Power function can be bounded via $P_{X, \Phi}(x) \leq C F(h)$. For the particular basis functions that we investigate, the order of conditional positive definiteness and $F(h)$ are given in table 1. As a reference for the Sobolev splines we give [4]. The results for the 
compactly supported radial basis functions and explicit formulas can be found in $[14,15]$. The degree of the polynomial is minimal under the following conditions:

1) $\Phi(x)=\phi\left(\|x\|_{2}\right)$ is a compactly supported function which consists of a univariate polynomial within its support.

2) The function $\Phi$ is positive definite on $\mathbb{R}^{d}$ and the even extension of $\phi$ is in $C^{2 \ell}$.

\section{Approximation in Sobolev spaces}

We now turn to the investigation of the approximation error between $u$ as the solution of (2.4) and the discrete Rayleigh-Ritz solution $u_{N}$ coming from $V_{N}$, where $V_{N}$ is given by (3.1) belonging to a special positive definite function $\Phi$. As mentioned in the introduction, we assume $u$ to be somewhat more regular, say $u \in W_{2}^{k}(\Omega)$ with $k>\frac{d}{2}$. Furthermore, according to the $C^{1}$-smoothness of the boundary of $\Omega$ there is a continuous extension mapping $E: W_{2}^{k}(\Omega) \rightarrow W_{2}^{k}\left(\mathbb{R}^{d}\right)$ (cf. [3]), and we will denote the extended function $E u \in W_{2}^{k}\left(\mathbb{R}^{d}\right)$ by $u$ again. This allows us to use the theory of radial basis functions and to identify $W_{2}^{k}\left(\mathbb{R}^{d}\right)$ with the native space $\mathcal{F}_{\Phi}$ to a radial basis function $\Phi \in L_{1}\left(\mathbb{R}^{d}\right)$ with Fourier transform $\widehat{\Phi}$ having the property

$$
c_{1}\left(1+\|\omega\|_{2}\right)^{-2 k} \leq \widehat{\Phi}(\omega) \leq c_{2}\left(1+\|\omega\|_{2}\right)^{-2 k}
$$

with positive constants $c_{1}, c_{2}$. This property will be abbreviated by

$$
\widehat{\Phi}(\omega) \sim\left(1+\|\omega\|_{2}\right)^{-2 k} .
$$

Following Cea's lemma (2.6), we have to bound

$$
\inf _{v \in V_{N}}\|u-v\|_{W_{2}^{1}(\Omega)}
$$

in terms of $h$ as defined in (3.3).

Theorem 4.1. Let $\Omega \subseteq \mathbb{R}^{d}$ be an open and bounded domain, having a $C^{1}$-boundary. Denote by $s_{u}$ the interpolant on $X=\left\{x_{1}, \ldots, x_{N}\right\} \subseteq \Omega$ to a function $u \in W_{2}^{k}(\Omega)$ with $k>d / 2$. Then there exists a constant $h_{0}>0$ such that for all $X$ with $h \leq h_{0}$, where $h$ is defined by (3.3), the estimate

$$
\left\|u-s_{u}\right\|_{W_{2}^{j}(\Omega)} \leq C h^{k-j}\|u\|_{W_{2}^{k}(\Omega)}
$$

is valid for $0 \leq j \leq k$.

Proof. Let us first assume $0 \leq j \leq k-\frac{d}{2}$. Since $k>\frac{d}{2}$, this covers, in particular, the case $j=0$. The function $u \in W_{2}^{k}(\Omega)$ can be extended to a function $E_{\Omega} u \in W_{2}^{k}\left(\mathbb{R}^{d}\right)$, and the extension $E_{\Omega}$ is continuous. Combining this with the results from [17], we derive for all $\alpha \in \mathbb{N}_{0}^{d}$ with $|\alpha|<k-\frac{d}{2}$ and for all $x \in \mathbb{R}^{d}$ the estimate

$$
\left|D^{\alpha} u(x)-D^{\alpha} s_{u}(x)\right| \leq C P_{X, \Phi}^{(\alpha)}(x)\|u\|_{W_{2}^{k}\left(\mathbb{R}^{d}\right)} .
$$

The Power function $P_{X, \Phi}^{(\alpha)}(x)$ can be bounded from above in the following manner. There exists an $h_{1}$ such that for all $X$ with $h \leq h_{1}$ and all $x \in \Omega$ the estimate

$$
\left|P_{X, \Phi}^{(\alpha)}(x)\right| \leq C h^{k-\frac{d}{2}-|\alpha|}
$$

is valid. Here, $C$ denotes a constant independent of $x$ and $X$.

Now, we form $u^{B}:=E_{B}\left(E_{\Omega} u \mid B\right) \in W_{2}^{k}\left(\mathbb{R}^{d}\right)$ for a ball $B \subseteq \mathbb{R}^{d}$. It is possible to choose the extension mapping $E_{B}$ in such a way that the constant $C$ in 
$\left\|E_{B} u\right\|_{W_{2}^{k}\left(\mathbb{R}^{d}\right)} \leq C\|u\|_{W_{2}^{k}(B)}$ is independent of the radius and the position of the ball $B$ (cf. [15]). Thus (4.3) leads to

$$
\left\|D^{\alpha} u-D^{\alpha} s_{u}\right\|_{L_{2}(B)} \leq C \operatorname{vol}(B)^{\frac{1}{2}}\left\|P_{X, \Phi}^{(\alpha)}\right\|_{L_{\infty}(B)}\left\|u^{B}\right\|_{W_{2}^{k}\left(\mathbb{R}^{d}\right)} .
$$

According to [5] there exist $M, M_{1}, h_{2}>0$ and for $h \leq h_{2}$ a finite subset $T_{h} \subseteq \Omega$ such that the balls $B(t, h)$ and $B(t, M h)$ with radii $h$ and $M h$, respectively, centered at $t \in T_{h}$, satisfy

$$
B(t, h) \subseteq \Omega \subseteq \bigcup_{t \in T_{h}} B(t, M h)
$$

and such that $\sum_{t \in T_{h}} \chi_{B(t, M h)} \leq M_{1}$. Here $\chi_{A}$ denotes the characteristic function of the set $A$. This leads to

$$
\begin{aligned}
\left\|D^{\alpha} u-D^{\alpha} s_{u}\right\|_{L_{2}(\Omega)}^{2} & \leq \sum_{t \in T_{h}}\left\|D^{\alpha} u-D^{\alpha} s_{u}\right\|_{L_{2}(B(t, M h))}^{2} \\
& \leq C h^{d}\left\|P_{X, \Phi}^{(\alpha)}\right\|_{L_{\infty}\left(\Omega^{*}\right)}^{2} \sum_{t \in T_{h}}\left\|u^{B(t, M h)}\right\|_{W_{2}^{k}\left(\mathbb{R}^{d}\right)}^{2} \\
& \leq C h^{d}\left\|P_{X, \Phi}^{(\alpha)}\right\|_{L_{\infty}\left(\Omega^{*}\right)}^{2}\|u\|_{W_{2}^{k}\left(\mathbb{R}^{d}\right)}
\end{aligned}
$$

for $h \leq h_{2}$, where $\Omega^{*}:=\bigcup_{t \in T_{h}} B(t, M h)$. If we choose $h \leq h_{1}$ so small that also $(M+1) h \leq h_{0}$, we find for all $x \in \Omega^{*}$ certain points $t \in T_{h}$ and $x_{j} \in X$ such that we have $\|x-t\|_{2} \leq M h$ and $\left\|t-x_{j}\right\|_{2} \leq h$, which means that $\left\|x-x_{j}\right\|_{2} \leq(M+1) h$. Thus we can use (4.4) on $\Omega^{*}$ with $(M+1) h$ instead of $h$. But as $M$ does not depend on $h$ we get

$$
\left\|D^{\alpha} u-D^{\alpha} s_{u}\right\|_{L_{2}(\Omega)} \leq C h^{k-|\alpha|}\|u\|_{W_{2}^{k}(\Omega)}
$$

for $|\alpha|<k-d / 2$ and sufficiently small $h$, using the continuity of $E_{\Omega}$ again. This means that

$$
\left\|u-s_{u}\right\|_{W_{2}^{j}(\Omega)} \leq C h^{k-j}\|u\|_{W_{2}^{k}(\Omega)}
$$

for $0 \leq j<k-\frac{d}{2}$. For the remaining case $k-\frac{d}{2} \leq j \leq k$ we use the fact that $s_{u}$ is already an element of $W_{2}^{k}\left(\mathbb{R}^{d}\right)$. This leads to

$$
\begin{aligned}
\left\|u-s_{u}\right\|_{W_{2}^{k}(\Omega)}^{2} & =\left\|E_{\Omega} u-s_{u}\right\|_{W_{2}^{k}(\Omega)}^{2} \\
& \leq\left\|E_{\Omega} u-s_{u}\right\|_{W_{2}^{k}\left(\mathbb{R}^{d}\right)}^{2} \\
& \leq C\left|E_{\Omega} u-s_{u}\right|_{\Phi}^{2} \\
& \leq C\left|E_{\Omega} u\right|_{\Phi}^{2} \\
& \leq C\left\|E_{\Omega} u\right\|_{W_{2}^{k}\left(\mathbb{R}^{d}\right)}^{2} \\
& \leq C\|u\|_{W_{2}^{k}(\Omega)}^{2},
\end{aligned}
$$

if we use the fact that $s_{u}=s_{E_{\Omega} u}$ is the best approximation to $E_{\Omega} u$ from $V_{N}$ with respect to $(\cdot, \cdot)_{\Phi}$. Thus we have proven the case $j=k$. As we already know the estimate for $j=0$, we can invoke an interpolation theorem [1]

$$
|u|_{W_{2}^{j}(\Omega)} \leq C\left\{\varepsilon^{-j}\|u\|_{L_{2}(\Omega)}+\varepsilon^{k-j}|u|_{W_{2}^{k}(\Omega)}\right\}
$$


with the Sobolev semi-norm $|u|_{W_{2}^{j}(\Omega)}^{2}:=\sum_{|\alpha|=j}\left\|D^{\alpha} u\right\|_{L_{2}(\Omega)}^{2}$ to get

$$
\begin{aligned}
\left|u-s_{u}\right|_{W_{2}^{j}(\Omega)} & \leq C\left\{\varepsilon^{-j} h^{k}\|u\|_{W_{2}^{k}(\Omega)}+\varepsilon^{k-j}\|u\|_{W_{2}^{k}(\Omega)}\right\} \\
& \leq C h^{k-j}\|u\|_{W_{2}^{k}(\Omega)}
\end{aligned}
$$

with $\varepsilon=h$. Summing up the semi-norms, we get the stated estimate.

Corollary 4.2. If $u \in W_{2}^{k}(\Omega), k>d / 2$, is the solution to the variational problem (2.4) and $u_{N} \in V_{N}$ is the solution of (2.5), where $V_{N}$ belongs to an $X$ satisfying $h \leq h_{0}$, then the error can be bounded by

$$
\left\|u-u_{N}\right\|_{W_{2}^{1}(\Omega)} \leq C h^{k-1}\|u\|_{W_{2}^{k}(\Omega)} .
$$

Proof. We use Cea's lemma in the form (2.6) to get

$$
\begin{aligned}
\left\|u-u_{N}\right\|_{W_{2}^{1}(\Omega)} & \leq C \inf _{v \in V_{N}}\|u-v\|_{W_{2}^{1}(\Omega)} \\
& \leq C\left\|u-s_{u}\right\|_{W_{2}^{1}(\Omega)} \\
& \leq C h^{k-1}\|u\|_{W_{2}^{k}(\Omega)}
\end{aligned}
$$

So far the radial basis function interpolant has to be formed with a specific $\Phi$ satisfying (4.1). In the next section we will pay attention to more general basis functions. These basis functions have to possess a (generalized) Fourier transform with a faster decay than given in (4.1).

\section{Approximation using general Basis functions}

A disadvantage in the application of the results of the last section is that the basis function $\Phi$ and the spaces $V_{N}$ have to be chosen as functions of the smoothness of the unknown solution $u$. But since this smoothness is unknown in general, we have to look for convergence results where $\Phi$ can be chosen independent of the smoothness of the solution. Therefore we still assume $u$ to be an element of $W_{2}^{k}(\Omega)$, and thus by extension of $W_{2}^{k}\left(\mathbb{R}^{d}\right)$, but take $u_{N}$ from a $V_{N}$ formed with a basis function that generates not the whole $W_{2}^{k}\left(\mathbb{R}^{d}\right)$ as its native space, but a smaller space. This means that we put more regularity into $\Phi$ than we assume for $u$. It will turn out that in this setting the same convergence results can be achieved as in the last section.

From now on let us denote the basis function $\Phi$ appearing in (4.1) by $\Phi_{0}$. This function generates the space $\mathcal{F}_{\Phi_{0}}=W_{2}^{k}\left(\mathbb{R}^{d}\right)$ as before. It will turn out that we now have to assume at least $k>\frac{d}{2}+1$ to bound the $W_{2}^{1}(\Omega)$-error. The function $\Phi_{1}$ which generates the subspaces $V_{N}$ is supposed to be "smoother" than $\Phi_{0}$ or, to be more precise, to satisfy $\mathcal{F}_{\Phi_{1}} \subseteq \mathcal{F}_{\Phi_{0}}$. Thus we have to investigate the approximation property of $V_{N}$ in $\mathcal{F}_{\Phi_{0}}=W_{2}^{k}\left(\mathbb{R}^{d}\right)$. This was done for the $L_{\infty}$-error in [12] and we are going to carry this over to our purpose. We start our investigation by chopping off the Fourier transform.

Lemma 5.1. Let $\Phi_{0}$ be given, such that $\widehat{\Phi}_{0}$ satisfies (4.1) with $k>m+d / 2$. For $u \in \mathcal{F}_{\Phi_{0}}$ we define the function $u_{M}$ by its Fourier transform $\widehat{u}_{M}:=\widehat{u} \chi_{M}$, where $\chi_{M}$ denotes the characteristic function of the ball centered at zero with radius $M$. Then for all $\alpha \in \mathbb{N}_{0}^{d}$ with $|\alpha| \leq m$ and all $x \in \mathbb{R}^{d}$ we have

$$
\left|D^{\alpha} u(x)-D^{\alpha} u_{M}(x)\right| \leq|u|_{\Phi_{0}} c_{0,|\alpha|}(M)
$$


with

$$
c_{0,|\alpha|}^{2}(M)=(2 \pi)^{-d} \int_{\|\omega\|_{2} \geq M}\|\omega\|_{2}^{2|\alpha|} \widehat{\Phi}_{0}(\omega) d \omega .
$$

Proof. The assumptions on $\Phi_{0}$ give $\mathcal{F}_{\Phi_{0}}=W_{2}^{k}\left(\mathbb{R}^{d}\right) \subseteq C^{m}\left(\mathbb{R}^{d}\right)$, and allow us to use the inverse Fourier transform for $u \in \mathcal{F}_{\Phi_{0}}$ to get

$$
D^{\alpha} u(x)=(2 \pi)^{-d} \int_{\mathbb{R}^{d}}(i \omega)^{\alpha} e^{i x^{T} \omega} \widehat{u}(\omega) d \omega,
$$

which leads to

$$
\begin{aligned}
& \left|D^{\alpha}\left(u-u_{M}\right)(x)\right| \leq(2 \pi)^{-d} \int_{\|\omega\|_{2} \geq M}\|\omega\|_{2}^{2|\alpha|} \widehat{u}(\omega) d \omega \\
& \quad \leq(2 \pi)^{-d}\left(\int_{\|\omega\|_{2} \geq M} \frac{|\widehat{u}(\omega)|^{2}}{\widehat{\Phi}_{0}(\omega)} d \omega\right)^{1 / 2}\left(\int_{\|\omega\|_{2} \geq M}\|\omega\|_{2}^{2|\alpha|} \widehat{\Phi}_{0}(\omega) d \omega\right)^{1 / 2} \\
& \quad \leq|u|_{\Phi_{0}} c_{0,|\alpha|}(M) .
\end{aligned}
$$

Now we make use of the fact that $u_{M}$ is an element of $\mathcal{F}_{\Phi_{1}}$ for $u \in \mathcal{F}_{\Phi_{0}}$ if the conditions of the following theorem are satisfied. The domain $\Omega$ is still supposed to have a $C^{1}$-boundary.

Theorem 5.2. Let $V_{N}$ be given by (3.1) using the basis function $\Phi_{1}$. Let $\Phi_{0}$ satisfy (4.1) with $k>m+d / 2$, and let $\widehat{\Phi}_{0} / \widehat{\Phi}_{1}$ be bounded in every ball centered at zero. Then for every $u \in W_{2}^{k}(\Omega)$ there exists a function $s \in V_{N}$ such that for every $x \in \Omega$ and every $\alpha \in \mathbb{N}_{0}^{d}$ with $|\alpha| \leq m$

$$
\begin{aligned}
\left|D^{\alpha} u(x)-D^{\alpha} s(x)\right| & \leq\left(c_{0,|\alpha|}(M)+C_{01}(M) P_{X, \Phi_{1}}^{(\alpha)}(x)\right)|u|_{\Phi_{0}} \\
& \leq C\left(c_{0,|\alpha|}(M)+C_{01}(M) P_{X, \Phi_{1}}^{(\alpha)}(x)\right)\|u\|_{W_{2}^{k}(\Omega)}
\end{aligned}
$$

with

$$
C_{01}^{2}(M):=\sup _{\|\omega\|_{2} \leq M} \frac{\widehat{\Phi}_{0}(\omega)}{\widehat{\Phi}_{1}(\omega)} .
$$

The function $s$ does not depend on $\alpha$.

Proof. We choose $s=s_{u_{M}}$ and get

$$
\begin{aligned}
\left|D^{\alpha}(u-s)(x)\right| & \leq\left|D^{\alpha}\left(u-u_{M}\right)(x)\right|+\left|D^{\alpha}\left(u_{M}-s_{u_{M}}\right)(x)\right| \\
& \leq c_{0,|\alpha|}(M)|u|_{\Phi_{0}}+P_{\Phi_{1}, X}^{(\alpha)}(x)\left|u_{M}\right|_{\Phi_{1}} .
\end{aligned}
$$

But by

$$
\left|u_{M}\right|_{\Phi_{1}}^{2}=(2 \pi)^{-d} \int_{\|\omega\|_{2} \leq M} \frac{|\widehat{u}(\omega)|^{2}}{\widehat{\Phi}_{0}(\omega)} \frac{\widehat{\Phi}_{0}(\omega)}{\widehat{\Phi}_{1}(\omega)} d \omega \leq|u|_{\Phi_{0}}^{2} C_{01}^{2}(M)
$$

we derive

$$
\left|D^{\alpha}(u-s)(x)\right| \leq\left(c_{0,|\alpha|}(M)+C_{01}(M) P_{\Phi_{1}, X}^{(\alpha)}(x)\right)|u|_{\Phi_{0}} .
$$

Finally, $|u|_{\Phi_{0}} \leq C\|u\|_{W_{2}^{k}(\Omega)}$ leads to the last inequality. 
The approximation order will now be achieved by bounding the term on the right side $\left(c_{0,|\alpha|}(M)+C_{01}(M) P_{\Phi_{1}, X}^{(\alpha)}(x)\right)$ by powers of $h$. This is done in two steps:

- Choose $M$ such that $C_{01}(M) P_{\Phi_{1}, X}^{(\alpha)}(x) \leq c_{0,|\alpha|}(M)$. This leads to the error bound $\left|D^{\alpha}(u-s)(x)\right| \leq 2 C c_{0,|\alpha|}\|u\|_{W_{2}^{k}(\Omega)}$.

- Give an upper bound for $c_{0,|\alpha|}(M)$.

Of course, this has to depend upon the basis functions $\Phi_{0}$ and $\Phi_{1}$. While $\Phi_{0}$ is determined by (4.1), $\Phi_{1}$ is the basis function in question. As $c_{0,|\alpha|}(M)$ only depends on $\Phi_{0}$, we can compute it:

$$
\begin{aligned}
c_{0,|\alpha|}^{2}(M) & \leq C \int_{\|\omega\|_{2} \geq M}\|\omega\|_{2}^{2|\alpha|}\left(1+\|\omega\|_{2}\right)^{-2 k} d \omega \\
& =C \int_{M}^{\infty} r^{2|\alpha|+d-1}(1+r)^{-2 k} d r \\
& =C M^{2|\alpha|+d-2 k}
\end{aligned}
$$

for $|\alpha| \leq m$. The last constant $C$ can be chosen independently of $\alpha$ and $M$.

As every basis function we have in mind has an algebraically decaying Fourier transform, we use functions $\Phi_{1}$ which generate smoother and more general Sobolev spaces, i.e. we assume that

$$
\widehat{\Phi}_{1}(\omega) \sim\left(1+\|\omega\|_{2}\right)^{-2 \beta} .
$$

In contrast to (4.1), $\beta$ need not be in $\mathbb{N}$. To ensure $\mathcal{F}_{\Phi_{1}} \subseteq \mathcal{F}_{\Phi_{0}}$ we have to require $\beta \geq k$. This leads to

$$
C_{01}^{2}(M)=\sup _{\|\omega\|_{2} \leq M}\left(1+\|\omega\|_{2}\right)^{2 \beta-2 k}=C M^{2(\beta-k)} .
$$

Theorem 5.3. Assume $u \in W_{2}^{k}(\Omega)$ and $\Phi_{1}$ satisfies (5.2) with $\beta \geq k>\frac{d}{2}+m$. Let $V_{N}$ be given by (3.1) using $\Phi_{1}$. Then there exists a function $s \in V_{N}$ such that for $x \in \Omega$ and $|\alpha| \leq m$

$$
\left|D^{\alpha} u(x)-D^{\alpha} s(x)\right| \leq C h^{k-|\alpha|}\|u\|_{W_{2}^{k}(\Omega)}
$$

if $h$ is sufficiently small. In particular, the estimate

is valid for $h \leq h_{0}$.

$$
\|u-s\|_{W_{2}^{m}(\Omega)} \leq C h^{k-m}\|u\|_{W_{2}^{k}(\Omega)}
$$

Proof. We extend $u$ to a function $u \in W_{2}^{k}\left(\mathbb{R}^{d}\right)=\mathcal{F}_{\Phi_{0}}$. According to Theorem 5.2 and (4.4) we have for $x \in \Omega$ and $|\alpha| \leq m$

$$
\begin{aligned}
\left|D^{\alpha}\left(u-s_{u_{M}}\right)(x)\right| & \leq|u|_{\Phi_{0}}\left(c_{0,|\alpha|}(M)+C_{01}(M) P_{\Phi_{1}, X}^{(\alpha)}(x)\right) \\
& \leq|u|_{\Phi_{0}}\left(c_{0,|\alpha|}(M)+C_{01}(M) C h^{\beta-\frac{d}{2}-|\alpha|}\right)
\end{aligned}
$$

with arbitrary $M>0$. Now we have to choose $M$ such that $C_{01}(M) C h^{\beta-\frac{d}{2}-|\alpha|} \leq$ $c_{0,|\alpha|}(M)$ for $|\alpha| \leq m$. Replacing $c_{0,|\alpha|}(M)$ and $C_{01}(M)$ by (5.1) and (5.3) respectively, we see that this is satisfied if $M \leq C / h$. Substituting this $M$, we get

$$
\begin{aligned}
\left|D^{\alpha}\left(u-s_{u_{M}}\right)(x)\right| & \leq 2 c_{0,|\alpha|}(M)|u|_{\Phi_{0}} \\
& \leq C h^{k-\frac{d}{2}-|\alpha|}|u|_{\Phi_{0}} \\
& \leq C h^{k-\frac{d}{2}-|\alpha|}\|u\|_{W_{2}^{k}(\Omega)} .
\end{aligned}
$$


Following the lines of the first part of the proof of theorem 4.1, we gain an additional factor $h^{d / 2}$ and derive the stated inequality.

Using Cea's Lemma, we get

Corollary 5.4. Under the assumptions of $u \in W_{2}^{k}(\Omega)$ and $\Phi_{1}$ satisfying (5.2) with $\beta \geq k>\frac{d}{2}+1$, the discretization error for $u_{N} \in V_{N}$ with $V_{N}$ from (3.1) formed with $\Phi_{1}$ can be bounded by

$$
\left\|u-u_{N}\right\|_{W_{2}^{1}(\Omega)} \leq C h^{k-1}\|u\|_{W_{2}^{k}(\Omega)}
$$

for sufficiently small $h$.

As $W_{2}^{k}(\Omega)$ is dense in $W_{2}^{1}(\Omega)$, standard arguments yield

Corollary 5.5. Let $\Phi_{1}$ satisfy the conditions of the last corollary. Let $V_{N}$ belong to a set of centers $X_{N}$ satisfying $h=h_{X_{N}, \Omega} \rightarrow 0$ for $N \rightarrow \infty$. Then the solutions $u_{N}$ converge to $u$ :

$$
\left\|u-u_{N}\right\|_{W_{2}^{1}(\Omega)} \rightarrow 0
$$

Finally, we have to check the condition on $\beta$ for the basis functions mentioned previously. The parameters refer to table 1 .

Corollary 5.6. Under the assumptions of theorem 5.3 and corollary 5.4,

$$
\left\|u-u_{N}\right\|_{W_{2}^{1}(\Omega)} \leq C h^{k-1}\|u\|_{W_{2}^{k}(\Omega)}
$$

for the choice of $\Phi_{1}$ as

- thin plate spline with $\mu \geq 2 k-d$,

- Sobolev spline with $\mu \geq k$,

- compactly supported functions with $\ell \geq k-\frac{d+1}{2}$.

Proof. The (generalized) Fourier transform $\widehat{\Phi}_{1}$ for thin plate splines, Sobolev splines, and compactly supported functions satisfies

$$
\widehat{\Phi}_{1}(\omega)=C\|\omega\|_{2}^{-d-\mu}, \widehat{\Phi}_{1}(\omega)=\left(1+\|\omega\|_{2}^{2}\right)^{-\mu}
$$

and

$$
\widehat{\Phi}_{1}(\omega) \sim\left(1+\|\omega\|_{2}\right)^{-d-2 \ell-1},
$$

respectively. This is well known for thin plate splines, and Sobolev splines, and can be found in [16] for the compactly supported function of minimal degree. Thus $\beta$ equals $(d+\mu) / 2, \mu, \ell+(d+1) / 2$, respectively. The condition $\beta \geq k$ gives the conditions on the parameters.

\section{Conclusion}

We have shown that our approach using radial basis functions leads to the same error bounds in the energy norm as the classical finite elements:

$$
\left\|u-u_{h}\right\|_{W_{2}^{1}(\Omega)} \leq C h^{k-1}\|u\|_{W_{2}^{k}(\Omega)}
$$

for $u \in W_{2}^{k}(\Omega), k>\frac{d}{2}$, if we use basis functions that generate $W_{2}^{k}\left(\mathbb{R}^{d}\right)$ as their native space. We also derive this approximation property for $k>\frac{d}{2}+1$ if we use smoother elements than necessary. Furthermore, our approach works in arbitrary space dimension. Using the technique of Nitsche, we can get approximation orders for estimates in the $L_{2}-$ norm, which are again the same as for classical finite elements. 


\section{ACKNOWLEDGMENTS}

Help in proofreading was provided by G. Lube and R. Schaback.

\section{REFERENCES}

1. R. Adams, Sobolev spaces, Academic Press, New York, 1975. MR 56:9247

2. T. Belytschko, Y. Krongauz, D. Organ, M. Fleming, P. Krysl, Meshless methods: an overview and recent developments, Computer Methods in Applied Mechanics and Engineering, special issue on Meshless Methods, vol 139 (1996), pp 3- 47.

3. S. Brenner, L. Scott, The Mathematical Theory of Finite Element Methods, Springer Verlag, New York, 1994. MR 95f:65001

4. J. G. Dix, R. O. Ogden, An interpolation scheme with radial basis in Sobolev spaces $H^{s}\left(\mathbb{R}^{n}\right)$, Rocky Mountain Journal of Mathematics, Vol. 24, No. 4 (1994), pp 1319 - 1337. MR 95k:41003

5. J. Duchon, Sur l'erreur d'interpolation des fonctions de plusieurs variables par les $D^{m}$-splines, R.A.I.R.O. Analyse numérique, Vol. 12, No. 4 (1978), pp 325 - 334. MR 80j:41052

6. N. Dyn, Interpolation and approximation by radial and related functions, in: Approximation Theory VI, Vol. 1, C. K. Chui, L. L. Schumaker, J. D. Ward eds., Academic Press, Boston, 1983, pp 211-234. MR 92d:41002

7. W. R. Madych, S. A. Nelson, Multivariate interpolation and conditionally positive definite fucntions, Approx. Theory and its Appl. 4.4 (1988), pp 77-89. MR 90e:41006

8. M. J. D. Powell, The theory of radial basis function approximation in 1990, in: Advances in Numerical Analysis Vol. 2, W. Light, ed., Clarendon Press, Oxford, 1992, pp 105 - 210. MR 95c: 41003

9. E. Prugovečki, Quantum Mechanics in Hilbert Space, Academic Press, New York, 1971. MR 58:14457

10. R. Schaback, Creating surfaces from scattered data using radial basis functions, in: Mathematical Methods for Curves and Surfaces, M. Daehlen, T. Lyche, L. L. Schumaker, eds., Vanderbilt University Press, Nashville, 1995, pp 477-496. MR 96g:65025

11. R. Schaback, Multivariate interpolation and approximation by translates of a basis function, in: Approximation Theory VIII, volume 1: Approximation and Interpolation, C. K. Chui, L. L. Schumaker, eds., Academic Press, Boston, 1995, pp 491 - 514. MR 98g:41036

12. R. Schaback, Approximation by radial basis functions with finitely many centers, Constructive Approximation 12 (1996), pp 331-340. MR 97d:41013

13. R. Schaback, Z. Wu, Operators on radial functions, J. of Computational and Applied Mathematics 73 (1996), pp 257-270. MR 97g:42002

14. H. Wendland, Piecewise polynomial, positive definite and compactly supported radial functions of minimal degree, Advances in Comp. Math. 4 (1995), pp 389 - 396. MR 96h:41025

15. H. Wendland, Sobolev-type error estimates for interpolation by radial basis functions, in: Surface Fitting and Multiresolution Methods, A. LeMéhauté, C. Rabut, L. L. Schumaker, eds., Vanderbilt University Press, Nashville, 1997, pp 337-344.

16. H. Wendland, Error estimates for interpolation by compactly supported radial basis functions of minimal degree, Journal of Approx. Theory 93 (1998), pp 258-272. CMP 98:11

17. Z. Wu, R. Schaback, Local error estimates for radial basis function interpolation of scattered data, IMA J. of Numerical Analysis 13 (1993), pp 13-27. MR 93m:65012

Institut Für Numerische Und Angewandte Mathematik, Universität Göttingen, Lotzestrasse 16-18, D-37083 Göttingen, Germany

E-mail address: wendland@math.uni-goettingen.de 